\title{
Comprehensive early intervention for patients with first-episode psychosis in Japan (J-CAP): study protocol for a randomised controlled trial
}

\author{
Shinsuke Koike ${ }^{1 *}$, Atsushi Nishida ${ }^{2,3^{*}}$, Syudo Yamasaki ${ }^{1,4}$, Kayo Ichihashi ${ }^{5}$, Sanae Maegawa ${ }^{6}$, Tatsunobu Natsubori ${ }^{1}$, \\ Hirohiko Harima ${ }^{3}$, Kiyoto Kasai ${ }^{1}$, Izumi Fujita ${ }^{5}$, Masanori Harada ${ }^{6}$ and Yuji Okazaki ${ }^{3}$
}

\begin{abstract}
Introduction: Comprehensive approaches for patients with psychotic symptoms play essential roles in the symptomatic and functional outcomes of patients, especially during disease onset. In Japan, the shortage of mental health services, particularly for outpatients, and community-based supports has been a major problem. The purpose of this trial is to investigate the effectiveness and affordability of 18-month comprehensive early intervention services for patients with first-episode psychosis compared with typical treatment.

Methods: This interventional, parallel, single-blinded (open but blinded raters trial) was effectively designed. The participants are patients with a diagnosis of F2 or F3 (International Classification of Disease, 10 th revision), with psychotic symptoms. The inclusion criteria were an age of 15-35 years, onset of psychotic symptoms within 5 years, first-episode psychosis, and residence in the catchment area of each site. Allocation will be conducted equally between case management and standard care groups. After enrollment, standard care will be provided for both groups, and community-based care to promote recovery for 18 months will be provided for the comprehensive approach group. The primary outcome will be the function domain of the global assessment of functioning scores at 18 months after enrollment. Data assessment will be performed at enrollment and 18, 36, and 60 months after enrollment. The target sample size will be 150, and registration will occur from March 1, 2011, to September 30, 2012.
\end{abstract}

Discussion: This trial will provide promising results about the effectiveness and cost-effectiveness of early intervention services in Japan to improve the quality and quantity of community mental health services.

Trial registration: This trial was registered in The University Hospital Medical Information Network Clinical Trials Registry (No. UMIN000005092).

\section{Introduction}

Comprehensive approaches for patients with schizophrenia play essential roles in their symptomatic and functional outcomes[1-4]. Particularly after the onset of psychosis, most patients and their families are extremely confused and distressed because of the conditions of patients and the lack of knowledge about their illnesses. Several randomized controlled trials (RCTs) have

\footnotetext{
* Correspondence: skoike-tky@umin.ac.jp; nishida-at@igakuken.or.jp 'Department of Neuropsychiatry, Graduate School of Medicine, The University of Tokyo, Bunkyo-ku, Tokyo, 113-8655, Japan

${ }^{2}$ Department of Psychiatry \& Behavioral Science, Tokyo Metropolitan Institute of Medical Science, Setagaya-ku, Tokyo, 156-8506, Japan

Full list of author information is available at the end of the article
}

suggested that intensive community-based care for patients with first-episode psychosis (FEP) improve patient symptoms[5,6] and functional outcomes such as relapse, readmission, dropout from services, and social and vocational functioning[5-8]. Furthermore, additional analyses of cost-effectiveness revealed that patients with FEP who received intensive care had better outcomes without increase costs[9]. On the basis of these results, the use of early intervention services (EIS) has increased, especially in England[1].

Japan has among the best medical services in the world (e.g., the longest average life expectancy and smallest perinatal mortality rate). However, the shortage of

\section{C) Biomed Central}


mental health services for outpatients has been a major problem. In Japan, there are approximately 350,000 psychiatric beds, and approximately 210,000 patients majorly diagnosed with schizophrenia remain in psychiatric hospitals for more than 1 year (Reported from the Minster of Health, Labour, and Welfare). One issue preventing the discharge of patients with severe mental illnesses from hospitals is the shortage of community mental health services with psychosocial approaches, including family support despite the vast medical resources of Japan[10]. To change this situation and broaden EIS based on community settings, an RCT is needed to confirm the clinical effectiveness and affordability of EIS compared with existing hospital-based mental health service systems in Japan. Additionally, these trials must examine whether these specialized community mental health services will sustain the functional recovery of patients even after they finish 18month support.

\section{Methods}

\section{Trial design}

This trial is designed with effectiveness, interventional, parallel, single-blinded (open but blinded raters) trial. The allocation of participants will be equal (1:1) between the comprehensive approach (CAP) and standard care (SC) groups. The entire trial design is illustrated in Figure 1. After enrollment, all participants will be provided standard care, and in the CAP group, comprehensive community-based care by an early intervention team will be provided for 18 months. Eighteen months after enrollment, all participants will be provided standard care only. The assessment of clinical data will be conducted at enrollment and 18 (first end point), 36 (second end point), and 60 months after enrollment (last end point). The target sample size will be 150, and registration will occur from March 1, 2011, to September 30, 2012. The last follow-up date will be September 30, 2017. This trial was registered in the University Hospital Medical Information Network Clinical

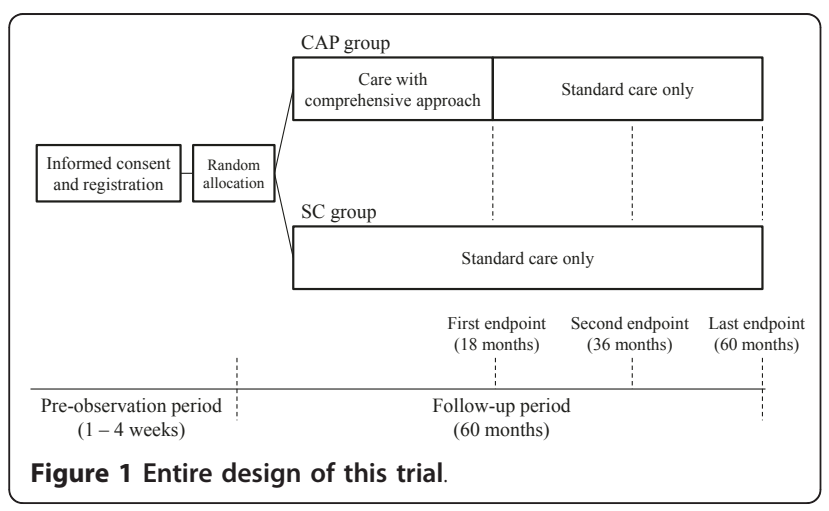

Trials Registry (UMIN-CTR) accepted from the International Committee of Medical Journal Editors (ICMJE) (No. UMIN000005092).

\section{Participants}

The participants are patients who received a diagnosis of F2 or F3 (International Classification of Disease, 10 th revision)[11], with psychotic symptoms, at 4 sites: the University of Tokyo Hospital, Tokyo Metropolitan Matsuzawa Hospital, Mie Prefectural Mental Medical Center, and Hinaga General Center for Mental Care and Sasagawa Clinic. The relevant details of the sites are summarized in Table 1.

Eligibility criteria are summarized in Table 2 . The inclusion criteria are an age of 15-35 years old, onset of psychotic symptoms within 5 years, first-episode psychosis, and residence in the catchment area of each site (Table 1). Psychotic symptoms are defined by the first clear evidence of a positive psychotic symptom (i.e., delusion, hallucination, or thought disorder) that was scored 4 or higher on the positive and negative symptom scales (PANSS) [12] regardless of its duration.

Exclusion criteria are premorbid IQ [13,14] of less than 80 , inability to sufficiently communicate in Japanese, requirement of care for any organic mental disorder or inpatient care for any physical condition, a history of dependency on alcohol and/or other substance of abuse, under physical restraint and/or seclusion, received electroconvulsive therapy and/or transcranial magnetic stimulation therapy within the past month, present involuntary hospitalization, not having been given the explanation of his or her condition or diagnosis from a psychiatrist, and being regarded as inappropriate by their doctors for any other reason. Although the use of illegal substances such as cannabis among young people has been a major problem, a very small number of young people have used these drugs in Japan. Therefore, we adopted a history of continuous substance abuse as one of the exclusion criteria in this trial.

All of the eligibility criteria will be assessed by psychiatrists at each site; to complement these criteria, the laboratory data of all patients will be assessed within 30 days of enrollment, and a brain CT or MRI will be performed within 12 months.

\section{Ethical consideration}

All participants will be presented with written informed consent to the ethical committee of each site (Univesity of Tokyo, No. 3307; Matsuzawa, No. 22-23; Mie, H23.2.21; Hinaga, H22.12.22) according to the Declaration of Helsinki after receiving a complete explanation of this trial. 
Table 1 Characteristics of facilities, number of patients, and number of staff members on early intervention teams at each site

\begin{tabular}{|c|c|c|c|c|c|}
\hline & & Univ of Tokyo & Matsuzawa & Mie & Hinaga \\
\hline \multicolumn{2}{|c|}{ Beds in closed wards } & 29 & 713 & 200 & 255 \\
\hline \multicolumn{2}{|c|}{ Beds in open wards } & 31 & 149 & 200 & 300 \\
\hline Annual & Total & 1107 & 2150 & 896 & 706 \\
\hline hospital & $\begin{array}{l}15-35 \text { years } \\
\text { old }\end{array}$ & 381 & 445 & 165 & 184 \\
\hline admissions & F2 & 129 & 212 & 55 & 94 \\
\hline \multicolumn{2}{|c|}{$\begin{array}{c}\text { Average number of daily } \\
\text { patients the in outpatient unit }\end{array}$} & 170 & 329.4 & 214.2 & 309.8 \\
\hline \multicolumn{2}{|c|}{$\begin{array}{l}\text { Average number of daily } \\
\text { participants in the outpatient } \\
\text { rehabilitation center }\end{array}$} & 33.1 & 57.6 & 49.9 & 109.6 \\
\hline \multirow{3}{*}{$\begin{array}{l}\text { Annual new } \\
\text { patients }\end{array}$} & Total & 1102 & 5656 & 1405 & 1150 \\
\hline & $\begin{array}{l}15-35 \text { years } \\
\text { old }\end{array}$ & 474 & NA & 476 & 450 \\
\hline & F2 & 124 & NA & 43 & 58 \\
\hline \multicolumn{2}{|c|}{ Catchment area } & $\begin{array}{l}\text { Bunkyo, Arakawa, } \\
\text { Taito, and Chiyoda }\end{array}$ & $\begin{array}{l}\text { Setagaya, Suginami, Ota, Meguro, } \\
\text { Shibuya, Komae, Chofu, and Mitaka }\end{array}$ & $\begin{array}{l}\text { Tsu, Matsuzaka, Suzuka, } \\
\text { Iga, Nabari, and Ise }\end{array}$ & $\begin{array}{c}\text { Yokkaichi, Suzuka, } \\
\text { Kameyama, and Mie-gun }\end{array}$ \\
\hline \multicolumn{2}{|c|}{ Population (× 1000) } & 800 & 3020 & 870 & 620 \\
\hline \multirow{6}{*}{$\begin{array}{l}\text { Members of } \\
\text { the EIS team }\end{array}$} & Psychiatrists & 6 & 5 & 1 & 3 \\
\hline & Nurses & 0 & 4 & 5 & 6 \\
\hline & Psychologists & 3 & 0 & 1 & 2 \\
\hline & $\begin{array}{l}\text { Psychiatric } \\
\text { social workers }\end{array}$ & 1 & 2 & 3 & 2 \\
\hline & $\begin{array}{c}\text { Vocational } \\
\text { workers }\end{array}$ & 0 & 0 & 1 & 1 \\
\hline & Pharmacist & 0 & 0 & 1 & 0 \\
\hline
\end{tabular}

Abbreviations: Univ of Tokyo, the University of Tokyo Hospital; Matsuzawa, Tokyo Metropolitan Matsuzawa Hospital; Mie, Mie Prefectural Mental Medical Center; Hinaga, Hinaga General Center for Mental Care and Sasagawa Clinic; ElS, early intervention service.

\section{Table 2 Summary of eligibility criteria}

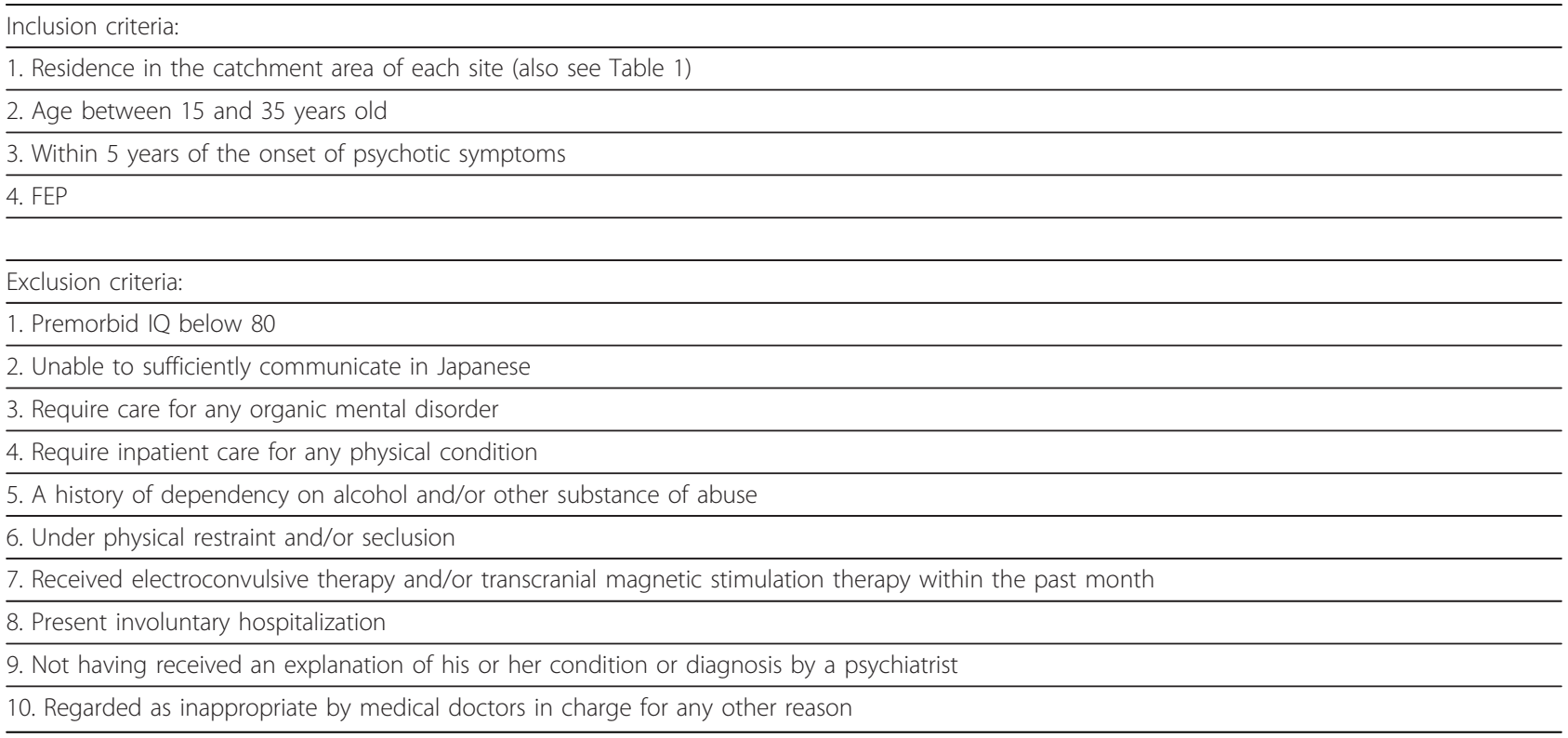




\section{Intervention}

For the CAP group, we will provide specialized early intervention teams by well-trained case managers and psychiatrists about their symptoms and daily activities for 18 months after enrollment. Case managers will promote participant recovery and social participation in cooperation with the early intervention team by using combination of cognitive behavioral therapeutic (CBT) approaches, psychoeducational approaches, family interventions, discharge support, and pharmacological therapy in accordance with the guidelines for FEP. The details of comprehensive community-based care are described later in the text. After 18 months, the participants will receive only standard care at each site. For the SC group, we will provide only standard care at each site throughout the entire study.

\section{CBT approach}

Several researches have suggested that CBT adapted to patients with early psychosis has effectiveness regarding their persistent positive symptoms, anxiety, and/or depression[15]. In this trial, we will not adopt structured CBT but needs-based CBT approaches in comprehensive community-based care. Case managers will receive basic CBT training and use the skills and techniques of CBT in comprehensive community-based care. The range and intensity of $\mathrm{CBT}$ will vary according to participant need as assessed by the early intervention team. Through CBT sessions, case managers will provide monitoring and coping skills for patients regarding their symptoms as well as functional skills for their daily lives.

\section{Psychoeducational approach}

Young people and their families rarely learn about symptoms, outcomes, and methods of coping with psychosis until they have mental illnesses; in other cases, they received incorrect information in addition to being subjected to discrimination and stigma. Therefore, they have significant stress and confusion because of the lack of information about psychosis. Several studies have suggested that psychoeducation is effective for relapse prevention, reducing hospital admission, and adherence of medication[16-18]. In this trial, the psychoeducational program for people with FEP will consist of sessions that provide not only information about etiology, symptoms, treatments, and outcome of psychosis, but also positive and helpful information toward functional recovery. In addition, we will provide psychoeducational programs for their families as described in the following section.

\section{Family intervention}

Family interventions will consist of 2 main domains of psychoeducation and family support for the family members of patients with mental illnesses. As described above, family members usually have little correct knowledge but a plethora of incorrect information and a great deal of stigma. Therefore, family members are also confused about methods of assisting patients who have recently exhibited psychotic symptoms. Several studies have suggested that family intervention for early psychosis is effective for relapse prevention, reducing hospital admission, and adherence of medication[19-21]. Family interventions for early psychosis will consist of psychoeducational programs and psychological therapy or counseling for families to relieve their burdens regarding the care of people with psychosis. Psychoeducational programs for families will consist of lectures about psychosis and peer group sessions. Psychological therapy and counseling for families will be provided through individual sessions.

\section{Discharge support}

More than 210,000 patients with schizophrenia stay in hospital for more than 1 year in Japan, mainly because of a lack of community mental health services[10]. This is particularly true for patients with psychosis for whom their family members are unable to obtain information and support from psychiatric community services. In this trial, case managers will encourage patients to live in their communities by introducing additional psychiatric community services, if needed.

\section{Pharmacological therapy in accordance with the guidelines} for FEP

Several pharmacological guidelines for schizophrenia and mood disorder are available worldwide. However, some psychiatrists in Japan have developed experimence-oriented strategies that result in polypharmacy for patients with schizophrenia[22]. Polypharmacy can exacerbate the negative symptoms and cognitive impairments of patients in addition to promoting dropped-out from treatment[22]. One reason why psychiatrists tend to prescribe more tablets may be that patients' treatment strategy and prescription are mainly decided by their psychiatrists who know little information except from the consultation with patients and their family members. In this trial, we will conduct regular meetings with early intervention teams and propose treatment and prescription strategies to psychiatrists according to the guidelines to eliminate unnecessary prescriptions.

\section{Strategy for replacement from EIS}

Recent studies about EIS for patients with FEP had negative results regarding the effectiveness of EIS discontinued in a longer term $[23,24]$. One reason may be that it is difficult to prepare patients to finish EIS and switch to normal services. On the basis of this result, we will emphasize "graduating from the EIS" and provide the policy of EI services to switch over to usual community services in order to easily maintain the effectives of EIS. Thus, we will also investigate whether this strategy for switch over will prolong the effectiveness of EIS. 


\section{Supervision for case management}

To standardize the intensity and quality of EIS, we will conduct training courses at least twice a year with required participation by case managers at each site, at which they will have to discuss their practices and cases. Supervision in meetings will be provided, and professionals and supervisors of other services will discuss and provide advice about their practices and cases.

\section{Outcomes and measurement items}

We will adopt the function domain of the global assessment of functioning (GAF-F) [25] scores at the first end point as the primary outcome measure. Secondary outcomes will be GAF-F at the second and last end points, symptom domain of global assessment of functioning (GAF-S), PANSS,[12] the World Health Organization quality of life 26-item version (WHO-QOL26),[26,27] brief evaluation of medication influences and beliefs (BEMIB),[28] care satisfaction of participants and their families, educational and vocational recovery rates, remission rate, re-admission rate, lost to follow-up rate, self-harm and suicide attempt rate, suicide rate, engagement behavior, and direct and indirect costs at each end point. All measurement items will also be assessed at baseline. We will record the presence of comorbid mental and physical disorders and relevant sociodemographic and clinical information about the participants and their families at baseline and at each end point. The details of the assessments are discussed in the following sections.

\section{GAF, GAF-F, and GAF-S}

GAF records the current objective symptomatic and functional conditions of participants on one analogous scale ranging from 0 (poor) to 100 (good)[25]. GAF-F rates the social and occupational functions of patients, and GAF-S rates their symptoms. GAF is adopted as the worse score of GAF-F and GAF-S. We will also use the modified GAF scale [29] to measure GAF, GAF-F, and GAF-S.

\section{PANSS}

PANSS records the current objective symptoms of patients on 30 items[12]. PANSS consists of 3 domains: positive symptoms, negative symptoms, and general psychopathology. Each item is rated from 1 (absent) to 7 (extreme), and the total score ranges from 30 to 210.

WHO-QOL26

WHO-QOL26 [26,27] measures the current subjective satisfaction of participants regarding their quality of life on 26 items. WHO-QOL26 consists of 4 domains: physical health, psychological health, social relationships, and environment. Each item is rated from 1 (poor) to 5 (good) and presented as an average score.

\section{BEMIB}

BEMIB measures the current drug adherence of participants to their medications on 8 items[28]. Each item is rated from 1 (completely disagree) to 5 (completely agree), and the total score ranges from 8 to 40 .

\section{Care satisfaction}

Care satisfaction will be measured by one simple item rated from 1 (very satisfied) to 4 (very dissatisfied). Participants and their families will provide subjective care satisfaction ratings after enrollment.

\section{Recovery rate}

Recovery will be measured by using the definition sheet adopted for EIS in England[30]. The item in the sheet termed "TRAINING AND OCCUPATION," which is rated from 0 (employment) to 4 (Not in Education, Employment or Training; NEET), will be assessed. For the initial assessment, this rating will be taken from the best occupational status achieved within the last 6 months for each end point taken from the point of last assessment.

\section{Remission rate}

We defined remission using a proposal from the Remission in Schizophrenia Working Group [31] that defined symptomatic remission of illness using 8 corresponding PANSS subscores (P1, P2, P3, N1, N4, N6, G5, and G9) of mild or less simultaneously on all items and for which remission regarding these scores was maintained for at least 6 months.

\section{Re-admission rate}

We will record all voluntary and involuntary admissions of participants at registration and during the follow-up period.

\section{Lost to follow-up rate}

Lost to follow-up will be defined at each end point as the refusal of further treatment despite the need and several attempts of reengagement (phone calls to patients and families in both groups and home visits to participants in the CAP group)[32]. We will consider the last successful contact the date of lost to follow-up.

\section{Self-harm, suicide attempt, and suicide rate}

Self-harm and suicide attempt will be measured using the items in the definition sheet "SELF-HARM and SUICIDE" on a scale of 0 (None) to 4 (Severe problems) [30]. We will assess the inquiry sheets for all of the suicide and severe self-harm actions at all sites.

\section{Engagement behavior}

Engagement, family engagement, and social relationships will be measured using the items in the definition sheet "ENGAGEMENT, FAMILY/CARER ENGAGEMENT, and RELATIONSHIPS" on a scale of 0 (Severe problems) to 4 (Very good) [30].

\section{Service costs and cost-effectiveness}

Service costs will be calculated using the Client Service Receipt Inventory (CSRI),[9,33], which can estimate service costs and cost-effectiveness, particularly in psychiatric contexts. On the basis of the CSRI, we will conduct interviews of daily living (e.g., service use, school, 
employment, and family conditions) at registration, at each end point, and if possible, at intermediate points between end points. Service costs will be calculated by the service costs and appropriate local unit costs. Costeffectiveness will be calculated by the combination of the outcomes related to the service costs and participant subjective quality of life assessments using WHOQOL26. Unavailable cost data will be estimated using other resources and models.

\section{Data reliability}

Some methodological problems regarding interrater reliability will exist. To control the quality of data assessment, we will conduct lectures for PANSS and GAF, including GAF-F and GAF-S, more than once a year using video movies to ensure accurate scoring. We will also calculate interrater reliability for PANSS and GAF scores provided by the raters in the lecture, and we will provide feedback for these results to maintain a high quality of assessment.

\section{Sample size}

The planned number of participants is 150 , as determined by previous RCT results, the clustering effects of each site, and the possibility of exhaustion of our resources. An RCT of EIS for patients with FEP in Holland indicated that EIS improved participant GAF-F scores in 24 months[5]. Another RCT of CBT in England revealed that $\mathrm{CBT}$ improved patient readmission rates and GAF scores in 18 months[6,7]. The effect sizes calculated from their results were $0.26,0.46$, and 0.58 , respectively. The estimated sample sizes using $G^{*}$ Power $3[34]$ (alpha error $=0.05$, beta error $=0.2$ ) were 370 , 150 , and 96 , respectively.

We will adopt 1 interim analysis and consider stopping the trial if the participants in the CAP group have unexpected effective outcomes compared with those in the SC group. We will conduct the interim analysis when half of the participants finish the trials until the first end point. Stopping rules will be planned on the basis of the O'Brien-Fleming method [35] for the GAF-F score, re-admission rate, lost to follow-up rate, selfharm and suicide attempt rate, and suicide rate at the first end point. We will consider stopping the trial on the basis of the stopping rule, baseline data, missing data, and site. Because of ethical issues, we will provide EIS to all participants until 18 months after allocation if the trial is halted.

\section{Randomization}

Enrollment and random allocation will be performed by central registration at the University Hospital Clinical Trial Alliance Clinical Research Supporting System (UHCT ACReSS) at the University of Tokyo. The managers of each site will enroll participants after examining their eligibility and informed consent. The type of allocation will be stratified and block randomization. We will adopt stratification as sites and inpatients/outpatients at enrollment. Owing to allocation concealment, the block size will be provided by UHCT ACReSS and will not be revealed to any researchers or staff until the end of the enrollment period. As this is a single-blinded trial, all assessments after enrollment will be conducted by independent raters with no knowledge of any treatment and care provided this trial.

\section{Statistical method}

All findings will be reported according to the revised CONSORT statement[36]. All analyses will be performed using SPSS 17.0 J (SPSS Inc., Chicago, IL, USA). All data will be analyzed under the intent-to-treat principle. The primary outcome will be analyzed using a simple Student's $t$ test and analyzed regarding potential confounders (e.g., age, gender, sociodemographic factors, site, and clinical characteristics) using regression models. Secondary outcomes will be analyzed using relevant tests at each end point and for possible confounders as described for the primary outcome. Subgroup analysis will be conducted at sites and performed for any possible confounders to differentiate the effectiveness of each situation and explore cluster effects.

\section{Discussion}

This trial has some possible limitations regarding sample size and the duration of case management. Previous studies of EIS for patients with FEP have suggested that although 1.5-year EIS improved patient social functions and readmission rates, these effects did not persist (5 years) $[23,24]$. However, these results also depend on the treatment provided to the control group, and community mental health services are widely available in Holland and England[1]. In the present situation in Japan, little psychological education is provided in schools and the workplace, and few mental health services are available in the community setting; consequently, much prejudice, discrimination, and stigma exists regarding psychosis[10]. In addition, we will emphasize "graduating from the service" in that case managers will continue to provide services after EIS after a participant enrolls in the trial. Therefore, this trial will demonstrate the effectiveness of EIS in Japan and also explore and consider exit strategies for EIS. Finally, the results of this trial will be used to inform policy makers and practitioners about the benefits, required human resources, and cost-effectiveness of EIS. This trial will provide helpful results about the effectiveness and cost-effectiveness of EIS in Japan to improve the quality and quantity of community mental services. 


\section{Acknowledgements}

We thank Motomu Suga and Tsuyoshi Araki in the University of Tokyo, Toru Wakejima in Tokyo Metropolitan Matsuzawa Hospital, Tomohisa Nakamura in Mie Prefectural Mental Medical Center, Kouhei Fujita and Tatsuya Tokura in Hinaga General Center for Mental Care and Sasagawa Clinic, and Syuntaro Ando in the Institute of Psychiatry, King's College London, for their advice and assistance with this trial. We thank Hiroshi Satonaka and Yoshihiro Arakawa in the Clinical Research Support Center, the University of Tokyo Hospital for use and assistance with registration and allocation system (UHCT ACReSS). We also thank Richard C. W. Hall for the permission of use of the modified GAF scale and translation into Japanese.

\section{Source of funding:}

This trial is funded by the Grants from the Ministry of Health, Labour, and Welfare (Health and Labour Sciences Research Grants, Research on Psychiatric and Neurological Diseases and Mental Health, H22-seishin-ippan015).

\section{Author details}

'Department of Neuropsychiatry, Graduate School of Medicine, The University of Tokyo, Bunkyo-ku, Tokyo, 113-8655, Japan. ${ }^{2}$ Department of Psychiatry \& Behavioral Science, Tokyo Metropolitan Institute of Medical Science, Setagaya-ku, Tokyo, 156-8506, Japan. ${ }^{3}$ Tokyo Metropolitan Matsuzawa Hospital, Setagaya-ku, Tokyo, 156-0057, Japan. ${ }^{4}$ Department of Rehabilitation, The University of Tokyo Hospital, Bunkyo-ku, Tokyo, 113-8655, Japan. ${ }^{5}$ Hinaga General Center for Mental Care and Sasagawa Clinic, Yokkaichi-shi, Mie, 510-8575, Japan. ${ }^{6}$ Mie Psychiatric Mental Care Center, Tsushi, Mie, 514-0818, Japan.

\section{Authors' contributions}

SK and AN equally contributed to design and management in this trial, and wrote most of the manuscript. SY made substantial contributions to conception and design of this trial and wrote the manuscript with regard to psychosocial care. KI, SM, TN, and HH made substantial contributions to conception and design of this trial. $\mathrm{KK}, \mathrm{IF}, \mathrm{MH}$, and $\mathrm{YO}$ are the directors at each site and made substantial contributions to the revision of the design and management in this trial. All authors read and approved the final manuscript.

Received: 15 May 2011 Accepted: 20 June 2011 Published: 20 June 2011

\section{References}

1. iFEVR: Meaningful lives: Supporting young people with psychosis in education, training and employment: an international consensus statement. Early Interv Psychiatry 2010, 4(4):323-326.

2. van Os J, Kapur S: Schizophrenia. Lancet 2009, 374(9690):635-645.

3. Barton GR, Hodgekins J, Mugford M, Jones PB, Croudace T, Fowler D: Cognitive behaviour therapy for improving social recovery in psychosis: cost-effectiveness analysis. Schizophr Res 2009, 112(1-3):158-163.

4. Patterson TL, Leeuwenkamp OR: Adjunctive psychosocial therapies for the treatment of schizophrenia. Schizophr Res 2008, 100(1-3):108-119.

5. Petersen L, Jeppesen $P$, Thorup A, Abel MB, Ohlenschlaeger J, Christensen TO, Krarup G, Jorgensen P, Nordentoft M: A randomised multicentre trial of integrated versus standard treatment for patients with a first episode of psychotic illness. BMJ 2005, 331(7517):602..

6. Craig TK, Garety P, Power P, Rahaman N, Colbert S, Fornells-Ambrojo M, Dunn G: The Lambeth Early Onset (LEO) Team: randomised controlled trial of the effectiveness of specialised care for early psychosis. BMJ 2004, 329(7474):1067..

7. Garety PA, Craig TK, Dunn G, Fornells-Ambrojo M, Colbert S, Rahaman N, Read J, Power P: Specialised care for early psychosis: symptoms, social functioning and patient satisfaction: randomised controlled trial. $\mathrm{Br} J$ Psychiatry 2006, 188:37-45.

8. Bird V, Premkumar P, Kendall T, Whittington C, Mitchell J, Kuipers E: Early intervention services, cognitive-behavioural therapy and family intervention in early psychosis: systematic review. Br J Psychiatry 2010, 197:350-356

9. McCrone P, Craig TK, Power P, Garety PA: Cost-effectiveness of an early intervention service for people with psychosis. Br J Psychiatry 2010, 196(5):377-382.
10. Oshima I, Mino Y, Inomata Y: How many long-stay schizophrenia patients can be discharged in Japan? Psychiatry Clin Neurosci 2007, 61(1):71-77.

11. World Health Organization: International Statistical Classification of Diseases and Related Health Problems 10 th Revision 2003.

12. Kay SR, Fiszbein A, Opler LA: The positive and negative syndrome scale (PANSS) for schizophrenia. Schizophr Bull 1987, 13(2):261-276.

13. Uetsuki M, Matsuoka K, Kasai K, Araki T, Suga M, Yamasue H, Maeda K, Yamazaki S, Furukawa S, Iwanami A, et al: Estimation of Premorbid IQ by Shortened Version of JARTs in Schizophrenia. Seishin lgaku 2007, 49(1):17-23.

14. Matsuoka K, Uno M, Kasai K, Koyama K, Kim Y: Estimation of premorbid IQ in individuals with Alzheimer's disease using Japanese ideographic script (Kanji) compound words: Japanese version of National Adult Reading Test. Psychiatry Clin Neurosci 2006, 60(3):332-339.

15. Morrison AP, French P, Walford L, Lewis SW, Kilcommons A, Green J, Parker S, Bentall RP: Cognitive therapy for the prevention of psychosis in people at ultra-high risk: randomised controlled trial. Br J Psychiatry 2004, 185:291-297.

16. Bechdolf A, Knost B, Nelson B, Schneider N, Veith V, Yung AR, Pukrop R: Randomized comparison of group cognitive behaviour therapy and group psychoeducation in acute patients with schizophrenia: effects on subjective quality of life. Aust N Z J Psychiatry 2010, 44(2):144-150.

17. Rummel-Kluge C, Schuster T, Peters S, Kissling W: Partial compliance with antipsychotic medication is common in patients with schizophrenia. Aust N Z J Psychiatry 2008, 42(5):382-388.

18. Rummel-Kluge C, Pitschel-Walz G, Bauml J, Kissling W: Psychoeducation in schizophrenia-results of a survey of all psychiatric institutions in Germany, Austria, and Switzerland. Schizophr Bull 2006, 32(4):765-775.

19. Pharoah F, Mari J, Rathbone J, Wong W: Family intervention for schizophrenia. Cochrane Database Syst Rev 2010, , 12: CD000088.

20. Gleeson JF, Cotton SM, Alvarez-Jimenez M, Wade D, Crisp K, Newman B, Spiliotacopoulos D, McGorry PD: Family outcomes from a randomized control trial of relapse prevention therapy in first-episode psychosis. $J$ Clin Psychiatry 2010, 71(4):475-483.

21. Garety PA, Fowler DG, Freeman D, Bebbington P, Dunn G, Kuipers E: Cognitive-behavioural therapy and family intervention for relapse prevention and symptom reduction in psychosis: randomised controlled trial. Br J Psychiatry 2008, 192(6):412-423.

22. Lim DS, Kang MS, Jeong JA, Bae YS: Semi-mature DC are immunogenic and not tolerogenic when inoculated at a high dose in collageninduced arthritis mice. Eur J Immunol 2009, 39(5):1334-1343.

23. Gafoor R, Nitsch D, McCrone P, Craig TK, Garety PA, Power P, McGuire P: Effect of early intervention on 5-year outcome in non-affective psychosis. Br J Psychiatry 2010, 196(5):372-376.

24. Bertelsen $M$, Jeppesen $P$, Petersen $L$, Thorup A, Ohlenschlaeger J, le Quach P, Christensen TO, Krarup G, Jorgensen P, Nordentoft M: Five-year follow-up of a randomized multicenter trial of intensive early intervention vs standard treatment for patients with a first episode of psychotic illness: the OPUS trial. Arch Gen Psychiatry 2008, 65(7):762-771.

25. American Psychiatry Association: Diagnostic and Statistical Manual of Mental Disorders. 4 edition. Washington, D.C. U.S.A.: American Psychiatric Press; 1994.

26. Development of the World Health Organization WHOQOL-BREF quality of life assessment. The WHOQOL Group. Psychol Med 1998, 28(3):551-558

27. Tazaki M, Nakane Y: WHOQOL 26 Japanese Version Tokyo: Kaneko Shobo Press; 1997.

28. Dolder CR, Lacro JP, Warren KA, Golshan S, Perkins DO, Jeste DV: Brief evaluation of medication influences and beliefs: development and testing of a brief scale for medication adherence. J Clin Psychopharmacol 2004, 24(4):404-409.

29. Hall RC: Global assessment of functioning. A modified scale. Psychosomatics 1995, 36(3):267-275.

30. Early Intervention Yorkshire and Humber. [http://its-services.org.uk/ earlyintervention/resources/].

31. Andreasen NC, Carpenter WT, Kane JM, Lasser RA, Marder SR Weinberger DR: Remission in schizophrenia: proposed criteria and rationale for consensus. Am J Psychiatry 2005, 162(3):441-449.

32. Lambert M, Bock T, Schottle D, Golks D, Meister K, Rietschel L, Bussopulos A, Frieling M, Schodlbauer M, Burlon Met, et al: Assertive community treatment as part of integrated care versus standard care: a 12-month trial in patients with first- and multiple-episode schizophrenia 
spectrum disorders treated with quetiapine immediate release (ACCESS trial). J Clin Psychiatry 2010, 71(10):1313-1323.

33. Beecham J, Knapp M: Costing psychiatric interventions. In Measuring Mental Health Needs.. 2 edition. Edited by: G T. London: Royal College of Psychiatrists; 2001:200-224

34. Erdfelder E, Faul F, Buchner A: GPOWER: A general power analysis program. Behavior Research Methods, Instruments, \& Computers 1996, 28:1-11.

35. O'Brien PC, Fleming TR: A multiple testing procedure for clinical trials. Biometrics 1979, 35:549-556.

36. Schulz KF, Altman DG, Moher D: CONSORT 2010 Statement: updated guidelines for reporting parallel group randomised trials. BMC Med 2010, 8:18.

doi:10.1186/1745-6215-12-156

Cite this article as: Koike et al:: Comprehensive early intervention for patients with first-episode psychosis in Japan (J-CAP): study protocol for a randomised controlled trial. Trials 2011 12:156.

\section{Submit your next manuscript to BioMed Central} and take full advantage of:

- Convenient online submission

- Thorough peer review

- No space constraints or color figure charges

- Immediate publication on acceptance

- Inclusion in PubMed, CAS, Scopus and Google Scholar

- Research which is freely available for redistribution

Submit your manuscript at www.biomedcentral.com/submit 